\title{
Post Traumatic Dementia and Bipolar Disorder: About a Case Report
}

1 - Centro Hospitalar Universitário do Algarve, Portugal 2 - Centro Hospitalar de Trás-os-Montes e Alto Douro, Vila Real, Portugal 3 - Centro Hospitalar Vila Nova de Gaia/Espinho, Vila Nova de Gaia, Portugal

\section{Background and Aims}

Traumatic Brain Injuries (TBI) are lesions that cause permanent or temporary physical, cognitive and psychosocial disorders. Data shows that even single TBI have a longterm association with higher rates of neurodegenerative diseases as well as higher morbility and mortality rates.

\section{Case Report}

60 years old male, no psychiatric history until 2016 when he was involved in a car crash that caused multiple facial fractures. After the accident, several behavioral changes were noticed, specifically poor performance in everyday tasks and adynamia. Due to the symptomatology the patient turns to a psychiatry consultation where he is medicated with bupropion $150 \mathrm{mg} /$ day, which is increased to $300 \mathrm{mg} /$ day because there is no significant improvement of the symptoms.

After the increase of bupropion, the patient was taken to the Emergency Room Service (ERS) due to a behaviour modification, characterized by sexual disinhibition, inadequacy on clothes choice, irritability, lack of sleep due to excessive activity. He was hospitalized in a mental health care unit, where the drug regimen was replaced with the introduction of sertraline $50 \mathrm{mg}$ daily, sodium valproate $500 \mathrm{mg} 2$ times a day, olanzapine $10 \mathrm{mg}$ daily. The patient received a diagnosis of bipolar disease.

\section{Follow-up}

He was followed in psychiatry for about 4 months. During those months, his depressive symptoms gradually worsened, being assessed by Psychiatry with complaints of anxiety, low mood, social isolation, deficits in memory retrieval and sexual dysfunction. Familiars remarked upon the inability to perform tasks due to long periods of inaction and difficulty in retaining new information, as simple instructions for every day routine like going to buy groceries. The patient description of his state was coherent with apathy, indicating a lack of interest or concern about life and the world around him.

\section{Brain CT and MRI}

CT scan showed atrophy on the frontal lobe

MRI scan showed the presence of several points of gliosis on the frontal lobe

\section{Psychological Assessment}

MMSE: $28 / 30$

MoCA: $22 / 30$

FAB: $12 / 18$

\section{Diagnostic Hipotheses}

\# Traumatic Frontal Lobe Syndrome
\# Major Depressive Disorder
\# Adjustment disorder with depressed
mood
\# Bipolar Affective Disorder

\section{Discussion and Conclussion}

Considering the patient's initial symptomatology, the clinical picture was interpreted as a major depressive disorder, which was initially treated with bupropion without clinical improvement, and that after increasing the dose, the patient experienced a manic condition motivating his internment. Manic symptoms were not associated with bupropion and the patient was diagnosed with a bipolar affective disorder and medicated according to the condition. There was a significant improvement in manic symptoms, however the patient always maintained apathy. The maintenance of this symptom made us think of the frontal lobe involvement after the accident, which would be demonstrated after imaging and neuropsychological evaluation.

The discussion of differential diagnoses is essential because the clinical presentation of this case is not the most usual of dementia, and misdiagnosis can be attributed to inadequate treatment. The suspicion of dementia after $T B I$ is essential in cases where there is a history of trauma, even if it is not recent. 